\title{
Causal Relationships Between Social Isolation and Osteoarthritis: A Mendelian Randomization Study in European Population
}

\section{Cong Zheng \\ Min-hui $\mathrm{He}$ \\ Jian-rong Huang \\ Yi He}

Emergency Trauma Center, Fifth Affiliated Hospital of Guangzhou Medical University, Guangzhou, 510700, People's Republic of China
Correspondence: Yi He Emergency Trauma Center, Fifth Affiliated Hospital of Guangzhou Medical University, 62I Gangwan Road, Huangpu District, Guangzhou, 510700, People's Republic of China

Email heyibone2013@I26.com
Objective: We aimed to investigate the causal relationships between social isolation and osteoarthritis.

Methods: Publicly available genome-wide association study (GWAS) summary statistics of social isolation and osteoarthritis in European population were obtained from the Neale lab Consortium and the Medical Research Council-Integrative Epidemiology Unit (MRC-IEU) consortium, respectively. Single nucleotide polymorphisms (SNPs) associated with the traits were identified by $\mathrm{P}<5 \times 10^{-8}$ and linkage disequilibrium $\mathrm{r}^{2}<0.1$. Three Mendelian randomization (MR) methods including the inverse-variance weighted (IVW) method, weighted median estimator, and MR-Egger regression were used to assess the potential causal effect of social isolation on osteoarthritis and the potential causal effect of osteoarthritis on social isolation. Leave-one-out analysis and test of directional horizontal pleiotropy via MR-Egger regression were performed as sensitivity analyses.

Results: When evaluating the causal effect of social isolation on osteoarthritis, five SNPs (rs12364432, rs13291079, rs2352075, rs4958586, rs599550) significantly associated with social isolation were studied as instruments, and social isolation was found to be causally associated with increased risk of osteoarthritis (odds ratio [OR] 1.197 (95\% confidence interval (CI) 1.096-1.308) estimated by the IVW method). When evaluating the causal effect of osteoarthritis on social isolation, six SNPs (rs10405617, rs12133235, rs13107325, rs2290573, rs3771501, rs75621460) significantly associated with osteoarthritis were studied as instruments but no causal effect of osteoarthritis on social isolation was found (OR 1.104 (95\% CI 0.887-1.375) estimated by the IVW method). Consistent causal relationships were observed when estimated by the weighted median estimator and MR-Egger regression. Leave-one-out analysis and test of directional horizontal pleiotropy suggested the robustness of the above findings.

Conclusion: Social isolation is causally associated with osteoarthritis, and further work is needed to investigate the potential mechanisms.

Keywords: osteoarthritis, social isolation, Mendelian randomization, causality

\section{Introduction}

Social relationships are well-established determinants of both mental and physical health. ${ }^{1}$ As one of the social determinants of health, social isolation can generally be defined as a state of complete or near-complete lack of contact between an individual and society, ${ }^{2}$ although the way it was defined and measured might vary between studies. ${ }^{3-5}$ An increased prevalence of social isolation has been observed, especially among the 
elderly, which is heightened by the coronavirus disease 2019 (COVID-19) pandemic. ${ }^{6}$ Therefore, to understand the potential effects of social isolation on well-being is important in a post-COVID-19 world. ${ }^{7,8}$ So far, social isolation has been demonstrated as a risk factor for various conditions, including coronary artery disease, stroke, and cancers. ${ }^{9-12}$

As a rheumatic musculoskeletal disorder, osteoarthritis is another highly prevalent condition in the elderly which has a considerable impact on an individual patient (eg, pain and disability) and causes heavy economic burden. ${ }^{13}$ The association between osteoarthritis and social isolation has been investigated. A prospective observational study included about 2000 elderly individuals from six European countries and found clinical osteoarthritis increased the risk of social isolation. ${ }^{14}$ Intuitively, it is rather reasonable to speculate that osteoarthritis may have a causal effect on developing social isolation. However, given the nature of the observational study design, association does not imply causation. Considering that bidirectional associations are common between social determinants and health, ${ }^{15}$ reverse causality cannot be ruled out between osteoarthritis and social isolation. Obviously, it is important to investigate the potential causal relationships between osteoarthritis and social isolation, since social isolation has been widely adopted as a measure against the COVID-19 pandemic.

Methodologically, a well-conducted randomized controlled trial is an ideal tool to investigate potential causal relationships, while for social determinants, such as social isolation, it would be impracticable due to ethical concern. As an increasingly used observational epidemiological method, Mendelian randomization (MR) is an analytical method that uses genetic variants as instrumental variables to assess the causal effect between exposure and outcome. ${ }^{16,17}$ Similar to a natural experiment of randomization, when cells divide during meiosis, genetic variants in the populations have been randomly allocated, making MR less likely to be affected by confounding or reverse causation than conventional observational studies. ${ }^{17,18}$ In addition, compared to the traditional single sample MR method, as one of the statistical methods for MR, the two-sample MR method provides an opportunity to substantially increase the statistical power by incorporating data from multiple sources, in which two different study samples are used to estimate the instrument-exposure and instrument-outcome associations to estimate a causal effect of the exposure on the outcome. ${ }^{18,19}$ This method has been successfully applied to the investigation into the causal relationships between social isolation and Alzheimer's disease, ${ }^{20}$ while currently there are no available studies that use the MR method to study the causal relationships between social isolation and osteoarthritis. Thus, in the present study, we used the twosample MR method to explore the potential causality between social isolation and osteoarthritis.

\section{Materials and Methods}

\section{Data Sources}

Via the R package "TwoSampleMR" (version 0.5.6), we accessed to the publicly available genome-wide association study (GWAS) summary statistics provided by the Integrative Epidemiology Unit (IEU) GWAS database (http://gwas-api.mrcieu.ac.uk/, Medical Research Council (MRC), University of Bristol). ${ }^{21}$ As presented in Table 1, genetic variants associated with social isolation came from the Neale lab Consortium, which contained 58,752 cases and 273,511 controls. The total number of single nucleotide polymorphism (SNP) was 10,894,596; genetic variants associated with osteoarthritis from the MRC-IEU consortium, which contained 38,472 osteoarthritis patients, 424,461 controls, as well as $9,851,867$ SNPs.

\section{Genetic Variants Associated with Social Isolation and Osteoarthritis}

When evaluating the causal effect of social isolation on osteoarthritis, to select the SNPs associated with social isolation as instruments, we used the threshold of statistical significance as previous $\left(\mathrm{P}<5 \times 10^{-8}\right.$, linkage disequilibrium $\left.r^{2}<0.1\right){ }^{22}$ Similarly, when evaluating the causal effect of osteoarthritis on social isolation, we identified the SNPs associated with osteoarthritis as instruments using the same criteria $\left(\mathrm{P}<5 \times 10^{-8}\right.$, linkage disequilibrium $\left.\mathrm{r}^{2}<0.1\right)$.

\section{Estimation of the Causal Relationships}

Three MR methods were utilized to explore the causal relationships between social isolation and osteoarthritis (including the causal effect of social isolation on osteoarthritis, and the causal effect of osteoarthritis on social isolation). The inverse-variance weighted (IVW) method is the most classical statistical MR method, which uses a meta-analysis approach to combine the Wald ratio estimates of the causal effect obtained from different SNPs. ${ }^{21,23}$ The IVW method relies on the assumption that all the included SNPs are valid instrumental variables, ${ }^{23}$ so the weighted median estimator is used to further verify the causality estimated by the IVW method. Unlike the IVW method, the premise of the 
Table I Details of the Traits Used in the Study

\begin{tabular}{|c|c|c|}
\hline Trait & Social Isolation & Osteoarthritis ${ }^{\dagger}$ \\
\hline ID & ukb-a-239 & ukb-b-I 4486 \\
\hline Year & 2017 & 2018 \\
\hline Author & Neale & Ben Elsworth \\
\hline Consortium & Neale Lab & MRC-IEU $\neq$ \\
\hline Sex & Males and Females & Males and Females \\
\hline Population & European & European \\
\hline Unit & SD & SD \\
\hline No. SNP & $10,894,596$ & $9,851,867$ \\
\hline Sample size & 332,263 & 462,933 \\
\hline Build & HGI9/GRCh37 & HGI9/GRCh37 \\
\hline Category & NA & Binary \\
\hline No. case & 58,752 & 38,472 \\
\hline No. control & 273,511 & 424,461 \\
\hline Note & http://www.nealelab.is/uk-biobank & $\begin{array}{l}\text { Output from GWAS pipeline using Phesant derived } \\
\text { variables from UKBiobank }\end{array}$ \\
\hline
\end{tabular}

Notes: ‘'Self-reported osteoarthritis; ${ }^{\ddagger}$ Output from genome-wide association study (GWAS) pipeline using Phesant-derived variables from UK Biobank.

Abbreviations: ID, identity; MRC-IEU, Medical research council-Integrative Epidemiology Unit; SD, standard deviation; SNP, single nucleotide polymorphism; NA, not available.

weighted median estimator allows up to half of the SNPs are not valid instrumental instruments. ${ }^{24}$ We also use the MR-Egger regression to estimate the investigated causal relationships, which do not rely on non-zero mean pleiotropy. $^{25}$ To further examine the robustness of the estimated causal relationships, a leave-one-out analysis (which estimated the causal effect after excluding every single SNP) and a test of directional horizontal pleiotropy (via the intercept term of MR-Egger regression) were performed as sensitivity analyses. The $\mathrm{R}$ program (R Core Team (2019). R: A language and environment for statistical computing. R Foundation for Statistical
Computing, Vienna, Austria. URL https://www. R-project.org/) was used to conduct all the analyses.

\section{Results}

\section{Causal Effect of Social Isolation on Osteoarthritis}

When evaluating the causal effect of social isolation on osteoarthritis, five SNPs were included as instruments for social isolation (rs12364432, rs13291079, rs2352075, rs4958586, rs599550). As presented in Table 2, the variation in the associations with social isolation was $0.056 \%$ among

Table 2 Associations of the Included SNPs with Social Isolation (Exposure) and Osteoarthritis (Outcome)

\begin{tabular}{|c|c|c|c|c|c|c|c|c|c|c|}
\hline \multirow[t]{2}{*}{ SNP } & \multirow[t]{2}{*}{ EA } & \multirow[t]{2}{*}{ Chromosome Location } & \multicolumn{4}{|c|}{ Social Isolation (Exposure) } & \multicolumn{4}{|c|}{ Osteoarthritis (Outcome) } \\
\hline & & & EAF & $\beta$ & SE & $\mathbf{P}$ & EAF & $\beta$ & SE & $\mathbf{P}$ \\
\hline rsI2364432 & $A$ & 11 & 0.347 & -0.007 & 0.001 & $3.25 \times 10^{-11}$ & 0.346 & -0.001 & 0.001 & 0.160 \\
\hline rsI3291079 & C & 9 & 0.425 & -0.006 & 0.001 & $5.35 \times 10^{-9}$ & 0.423 & -0.001 & 0.001 & 0.110 \\
\hline rs2352075 & C & 2 & 0.117 & 0.009 & 0.001 & $4.40 \times 10^{-10}$ & 0.118 & 0.003 & 0.001 & $2.30 \times 10^{-3}$ \\
\hline rs4958586 & A & 5 & 0.632 & 0.006 & 0.001 & $1.01 \times 10^{-8}$ & 0.632 & 0.000 & 0.001 & 0.550 \\
\hline rs599550 & A & 18 & 0.848 & 0.008 & 0.001 & $3.08 \times 10^{-9}$ & 0.848 & 0.002 & 0.001 & 0.025 \\
\hline
\end{tabular}

Abbreviations: SNP, single nucleotide polymorphism; EA, effect allele; EAF, effect allele frequency; SE, standard error. 
these five SNPs. The F statistic is a commonly used indicator for evaluating the bias of weak instrumental variables. ${ }^{26}$ In the present study, the F statistic was 37 , greater than 10 to effectively avoid the bias caused by weak instrumental variables. Associations of the five SNPs with the outcome of osteoarthritis are also presented in Table 2.

The causal relationship between social isolation and osteoarthritis estimated by using each single SNP as the instrument is presented in Figure 1. When the multiple SNPs were used as an instrument for social isolation, social isolation was found to be causally associated with increased risk of osteoarthritis (odds ratio [OR] 1.197 per SD increase (95\% confidence interval (CI) 1.096-1.308) estimated by the IVW method). Consistent causal relationships were observed when estimated by the weighted median estimator, and MR-Egger regression (Table 3 and Figure 1). Figure 2 presents the relation between the effect sizes of the SNPsocial isolation association (SD units) and the SNP- osteoarthritis associations ( $\log$ OR) with standard error bars, in which the slopes of the lines correspond to causal estimates using each of the three different methods.

\section{Causal Effect of Osteoarthritis on Social Isolation}

When evaluating the causal effect of osteoarthritis on social isolation, six SNPs were included as instruments for social isolation (rs10405617, rs12133235, rs13107325, rs2290573, rs3771501, rs75621460). As presented in Table 4, the variation in the associations with social isolation was $0.062 \%$ among these six SNPs, and the $\mathrm{F}$ statistic was 46, which was also greater than 10 . Associations of the six SNPs with the outcome of social isolation are also presented in Table 4.

The causal relationship between osteoarthritis and social isolation estimated by using each single SNP as the instrument is presented in Figure 3. When the multiple SNPs were

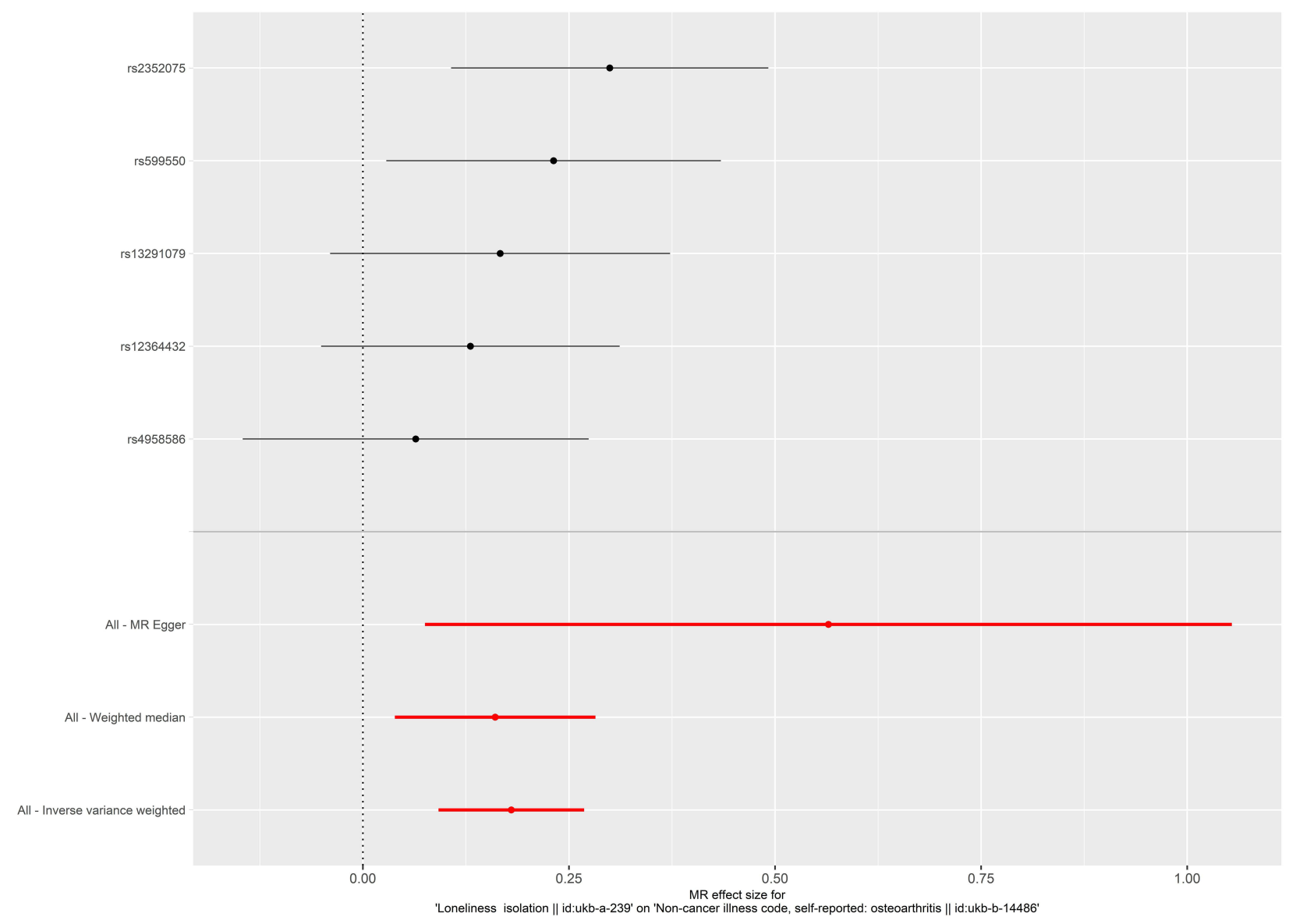

Figure I Forest plot of the causal effect of social isolation on osteoarthritis. Black points represent the log odds ratio for osteoarthritis per standard deviation increase in social isolation, which is produced by using each single nucleotide polymorphism (SNP) selected as a separate instrument. Red points show the combined causal estimate using all SNPs together as a single instrument, using the three different Mendelian randomization methods. Horizontal line segments denote $95 \%$ confidence intervals of the estimate. 
Table 3 Causal Effect of Social Isolation on Osteoarthritis

\begin{tabular}{|l|l|l|l|}
\hline Methods & $\boldsymbol{\beta}$ & SE & OR (95\% CI) \\
\hline IVW method & 0.180 & 0.045 & $\begin{array}{l}1.197 \\
(1.096-1.308)\end{array}$ \\
\hline Weighted median estimator & 0.160 & 0.058 & $\begin{array}{l}1.174 \\
(1.047-1.316)\end{array}$ \\
\hline MR-Egger regression & 0.565 & 0.250 & $\begin{array}{l}1.759 \\
(1.078-2.870)\end{array}$ \\
\hline
\end{tabular}

Abbreviations: SE, standard error; OR, odds ratio; $\mathrm{Cl}$, confidence interval; IVW inverse-variance weighted; MR, Mendelian randomization.

used as an instrument for osteoarthritis, no causal effect of osteoarthritis on social isolation was observed (OR 1.104 per SD increase, $95 \%$ CI $0.887-1.375$, estimated by the IVW method). Consistent results were found when estimated by the weighted median estimator, and MR-Egger regression (Table 5, Figures 3 and 4).

\section{Sensitivity Analysis}

The result of leave-one-out analysis indicated that the causal estimation was not biased or driven by any included SNP when evaluating the causal effect of social isolation on osteoarthritis (Figure 5). For the test of directional horizontal pleiotropy, the $\mathrm{P}$ value of the pleiotropy was relatively large, and the result was less likely to be affected by genetic pleiotropy (MR-Egger regression intercept= -0.003 , standard error $=0.002, \mathrm{P}=0.215$ ), suggesting the robustness of the above findings. Result of leave-one-out analysis for the evaluation of the causal effect of osteoarthritis on social isolation is presented in Figure 6.

\section{Discussion}

To the best of our knowledge, this is the first two-sample MR analysis to investigate the causal relationships between social isolation and osteoarthritis. This study

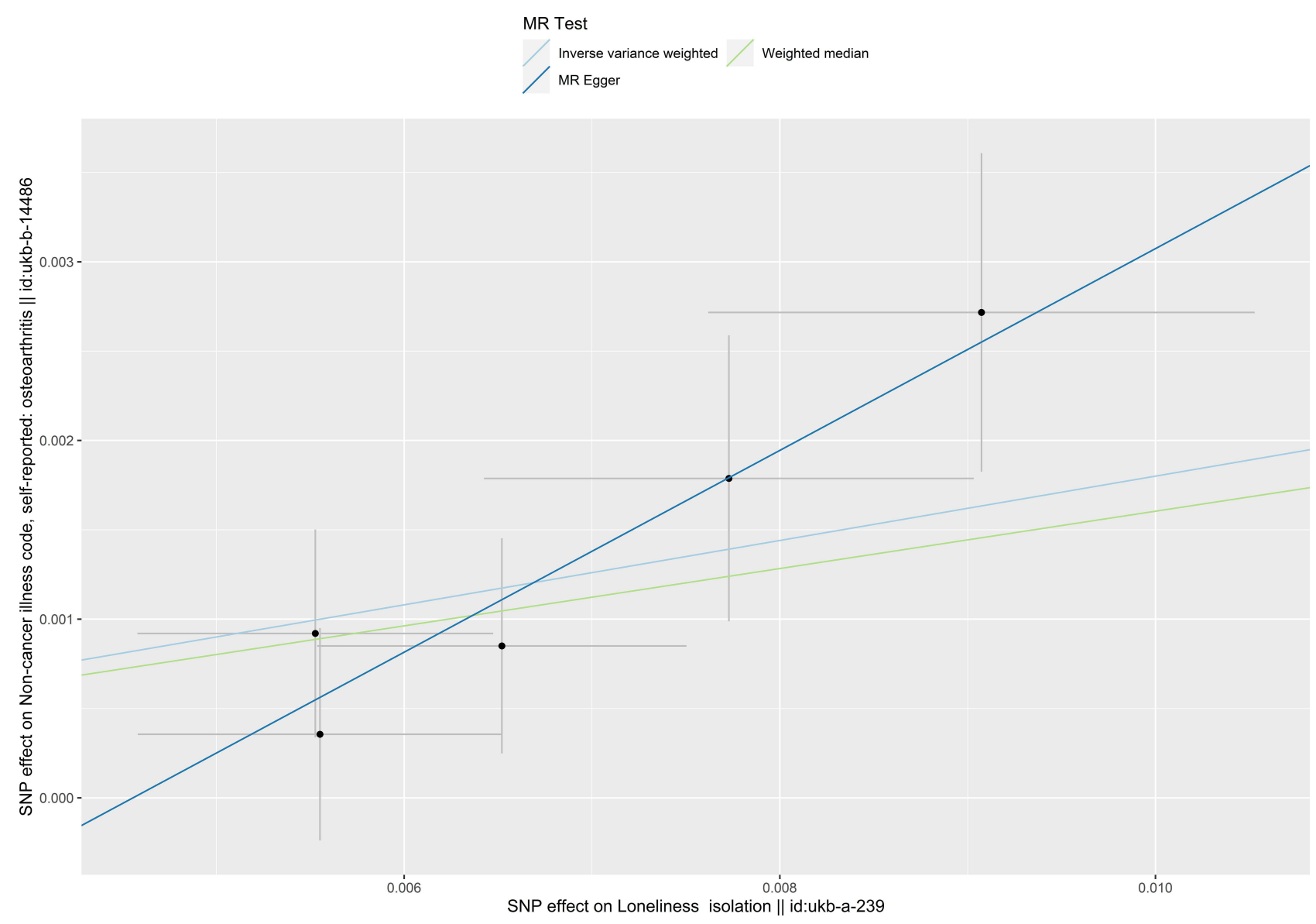

Figure 2 Scatter plot of the causal effect of social isolation on osteoarthritis. The plot presents the effect sizes of the single nucleotide polymorphism (SNP)-social isolation association ( $\mathrm{x}$-axis, standard deviation units) and the SNP-osteoarthritis association ( $y$-axis, log (odds ratio)) with $95 \%$ confidence intervals. The regression slopes of the lines correspond to causal estimates using the three Mendelian randomization methods. 
Table 4 Associations of the Included SNPs with Osteoarthritis (Exposure) and Social Isolation (Outcome)

\begin{tabular}{|c|c|c|c|c|c|c|c|c|c|c|}
\hline \multirow[t]{2}{*}{ SNP } & \multirow[t]{2}{*}{ EA } & \multirow[t]{2}{*}{ Chromosome Location } & \multicolumn{4}{|c|}{ Osteoarthritis (Exposure) } & \multicolumn{4}{|c|}{ Social Isolation (Outcome) } \\
\hline & & & EAF & $\boldsymbol{\beta}$ & SE & $\mathbf{P}$ & EAF & $\boldsymbol{\beta}$ & SE & $\mathbf{P}$ \\
\hline rs 10405617 & G & 19 & 0.664 & -0.003 & 0.001 & $4.30 \times 10^{-3}$ & 0.665 & -0.001 & 0.001 & 0.601 \\
\hline rs 12133235 & C & I & 0.421 & 0.003 & 0.001 & $1.20 \times 10^{-8}$ & 0.422 & -0.001 & 0.001 & 0.225 \\
\hline rs 13107325 & $\mathrm{~T}$ & 4 & 0.075 & 0.01 & 0.001 & $9.00 \times 10^{-14}$ & 0.075 & 0.003 & 0.002 & 0.052 \\
\hline rs2290573 & $A$ & 15 & 0.56 & 0.004 & 0.001 & $2.90 \times 10^{-6}$ & 0.567 & -0.001 & 0.001 & 0.448 \\
\hline rs377I50I & G & 2 & 0.524 & -0.004 & 0.001 & $1.40 \times 10^{-8}$ & 0.526 & -0.001 & 0.001 & 0.195 \\
\hline rs7562|460 & A & 19 & 0.027 & 0.013 & 0.002 & $1.20 \times 10^{-6}$ & 0.026 & -0.000 & 0.003 & 0.965 \\
\hline
\end{tabular}

Abbreviations: SNP, single nucleotide polymorphism; EA, effect allele; EAF, effect allele frequency; SE, standard error.

indicated that social isolation was causally associated with an increased risk of osteoarthritis. In contrast, osteoarthritis did not causally contribute to the level of social isolation. The main implication of the current study includes: (1) it establishes a causal relationship between social isolation and osteoarthritis; (2) given the global exposure to social isolation during the COVID-19 pandemic, the established causal relationship suggests there might be an increase in developing osteoarthritis after the pandemic, and efforts are warranted either for prevention, screening, or management; (3) the findings also warrant future studies on the underlying mechanism of the causal relationship

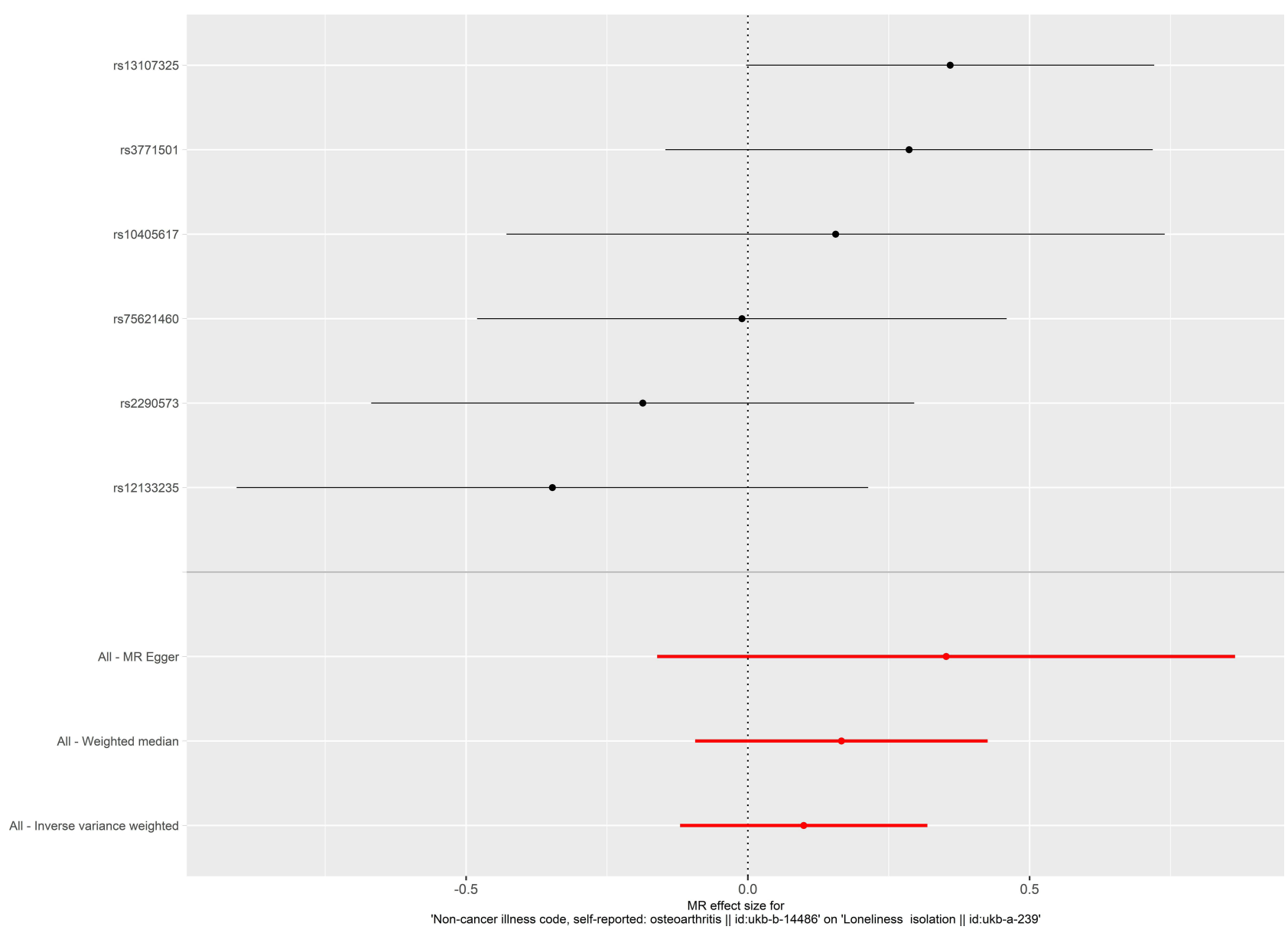

Figure 3 Forest plot of the causal effect of osteoarthritis on social isolation. Black points represent the log odds ratio for social isolation by osteoarthritis, which is produced by using each single nucleotide polymorphism (SNP) selected as a separate instrument. Red points show the combined causal estimate using all SNPs together as a single instrument, using the three different Mendelian randomization methods. Horizontal line segments denote $95 \%$ confidence intervals of the estimate. 
Table 5 Causal Effect of Osteoarthritis on Social Isolation

\begin{tabular}{|l|l|l|l|}
\hline Methods & $\boldsymbol{\beta}$ & SE & OR (95\% CI) \\
\hline IVW method & 0.099 & 0.112 & $\begin{array}{l}\text { I.I04 } \\
(0.887-I .375)\end{array}$ \\
\hline Weighted median estimator & 0.166 & 0.134 & $\begin{array}{l}\mathrm{I} .181 \\
(0.908-I .536)\end{array}$ \\
\hline MR-Egger regression & 0.352 & 0.262 & $\begin{array}{l}\mathrm{I} .422 \\
(0.85 I-2.374)\end{array}$ \\
\hline
\end{tabular}

Abbreviations: SE, standard error; OR, odds ratio; $\mathrm{Cl}$, confidence interval; IVW inverse-variance weighted; MR, Mendelian randomization.

between social isolation and osteoarthritis, which may also provide insight into novel target for management of osteoarthritis.

The main strength of our study is the MR study design, which helps to establish a causal relationship between social isolation and osteoarthritis. It has been well known that social isolation is harmful to both physical and psychological health. ${ }^{1,11,12}$ Previous observational studies have already established the association between social isolation and osteoarthritis. ${ }^{14,27}$ However, the traditional observational study designs are susceptible to interference from reverse causality and confounding factors, which limits the understanding of the relationship between social isolation and osteoarthritis. More importantly, different from general physical risk factors, the causal relationships between mental determinants and physical conditions are usually more complicated, which could be bidirectional. The development of MR methods, especially the twosample MR method, makes it easier and feasible to explore causal relationships in an observational way. To explore whether there was also a bidirectional effect between social isolation and osteoarthritis, we used the twosample MR method to evaluate the potential causal effect of social isolation on osteoarthritis, and the potential causal effect of osteoarthritis on social isolation, respectively. Such an investigation has not been reported before, which helps to fill in the knowledge gap in this topic. In addition,

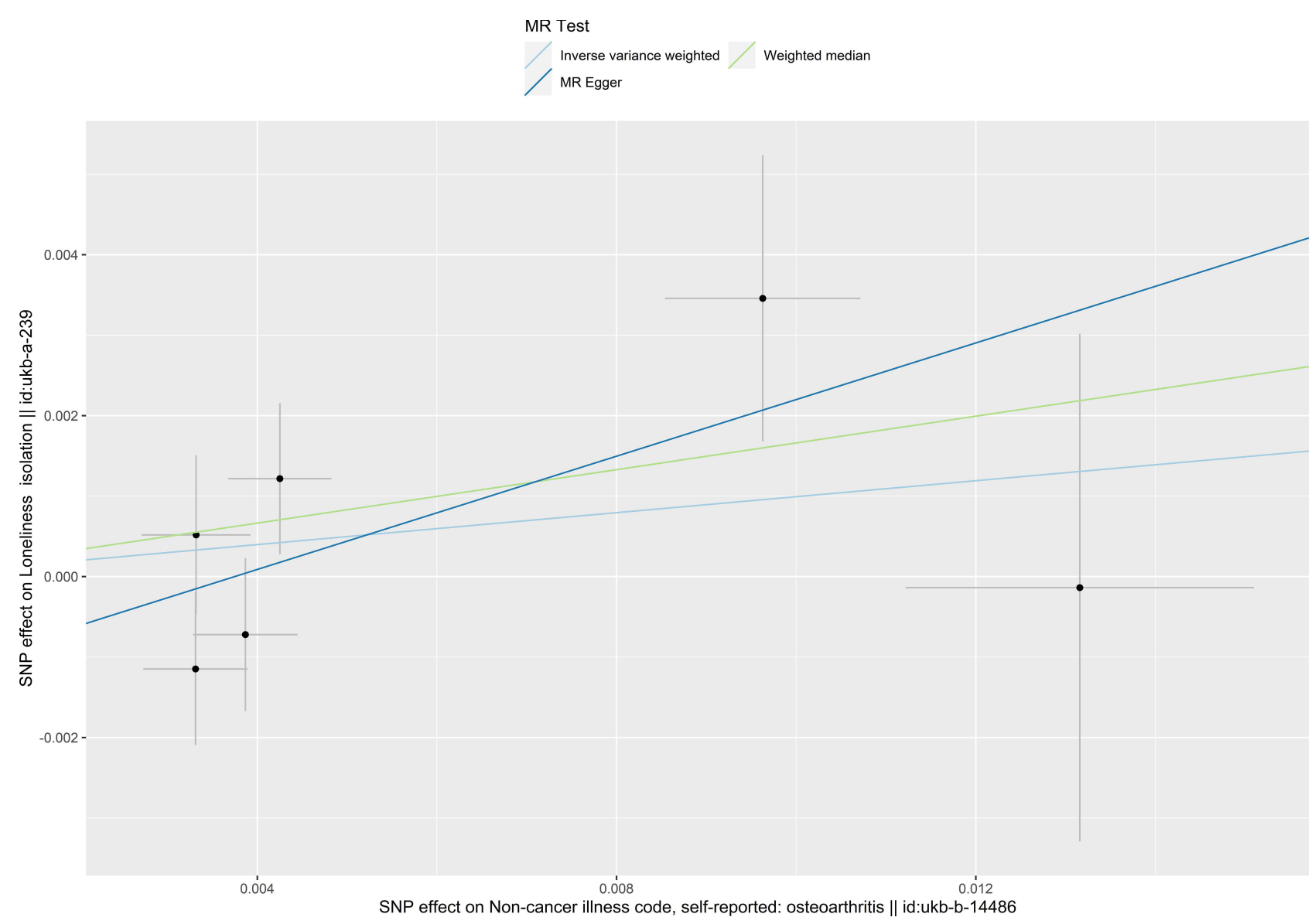

Figure 4 Scatter plot of the causal effect of osteoarthritis on social isolation. The plot presents the effect sizes of the single nucleotide polymorphism (SNP)-osteoarthritis association ( $\mathrm{x}$-axis, standard deviation units) and the SNP-social isolation association ( $y$-axis, log (odds ratio)) with $95 \%$ confidence intervals. The regression slopes of the lines correspond to causal estimates using the three Mendelian randomization methods. 


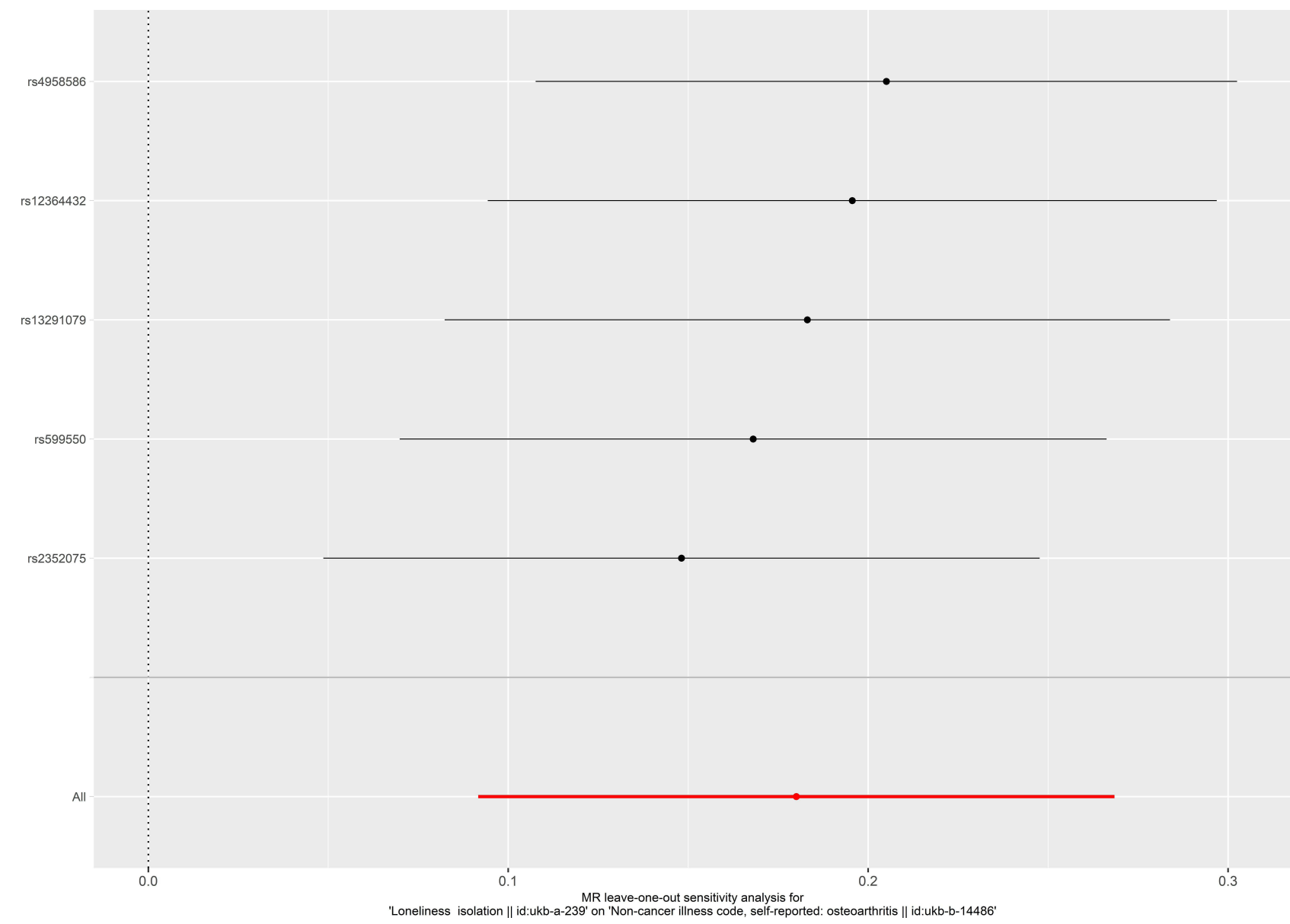

Figure 5 Leave-one-out analysis of the causal effect of social isolation on osteoarthritis. Each black point represents result of the inverse-variance weighted (IVW) method applied to estimate the causal effect of social isolation on osteoarthritis excluding each particular SNP from the analysis. Each red point depicts the IVW estimate using all SNPs. No single SNP is strongly driving the overall effect of social isolation on osteoarthritis in this leave-one-out sensitivity analysis.

in this study, we included large sample sizes through the two-sample MR approach, which helps to mitigate bias due to weak instrumental variables and provides the study with enough power to detect robust causality.

Several possible mechanisms may explain the causal effect of social isolation on osteoarthritis, in which the active inflammation status of individuals under social isolation may play a role. Elevated levels of proinflammatory cytokines have been observed in individuals under social isolation, including tumor necrosis factor alpha (TNF $\alpha$ ), and interleukin-6 (IL-6). ${ }^{28}$ Meanwhile, the level of IL-6 is associated with the degree of articular degeneration in osteoarthritis. ${ }^{29}$ Gut microbiota could be another possible pathway. The change of gut microbiota in osteoarthritis has attracted more and more attention from researchers, ${ }^{30,31}$ while it has been reported that psychosocial stress under social isolation leads to the activation of the hypothalamic pituitary adrenal axis and affects gut microbiota. ${ }^{32}$ Nevertheless, further studies are warranted since our study cannot directly provide evidence about the underlying mechanisms.

There were inevitably some limitations in our study. First, all the included participants were derived from European, so further research is needed to explore whether the finding is able to be generalizable to other populations. Second, both social isolation and osteoarthritis were based on the self-report data, rather than objective measurements, which might lead to measurement error. Third, due to the limitation of GWAS summary data, we were not able to conduct further subgroup analysis or get a more prominent view of how social isolation affected the occurrence and development of osteoarthritis. Fourth, as an inherent limitation of the two-sample MR method, sample overlap is possible in our study. Due to data limitation, we are unable to validate or improve this limitation. However, the large $F$ statistic for the instrument-exposure association in our study suggests that this concern can be minimized. ${ }^{33}$ 


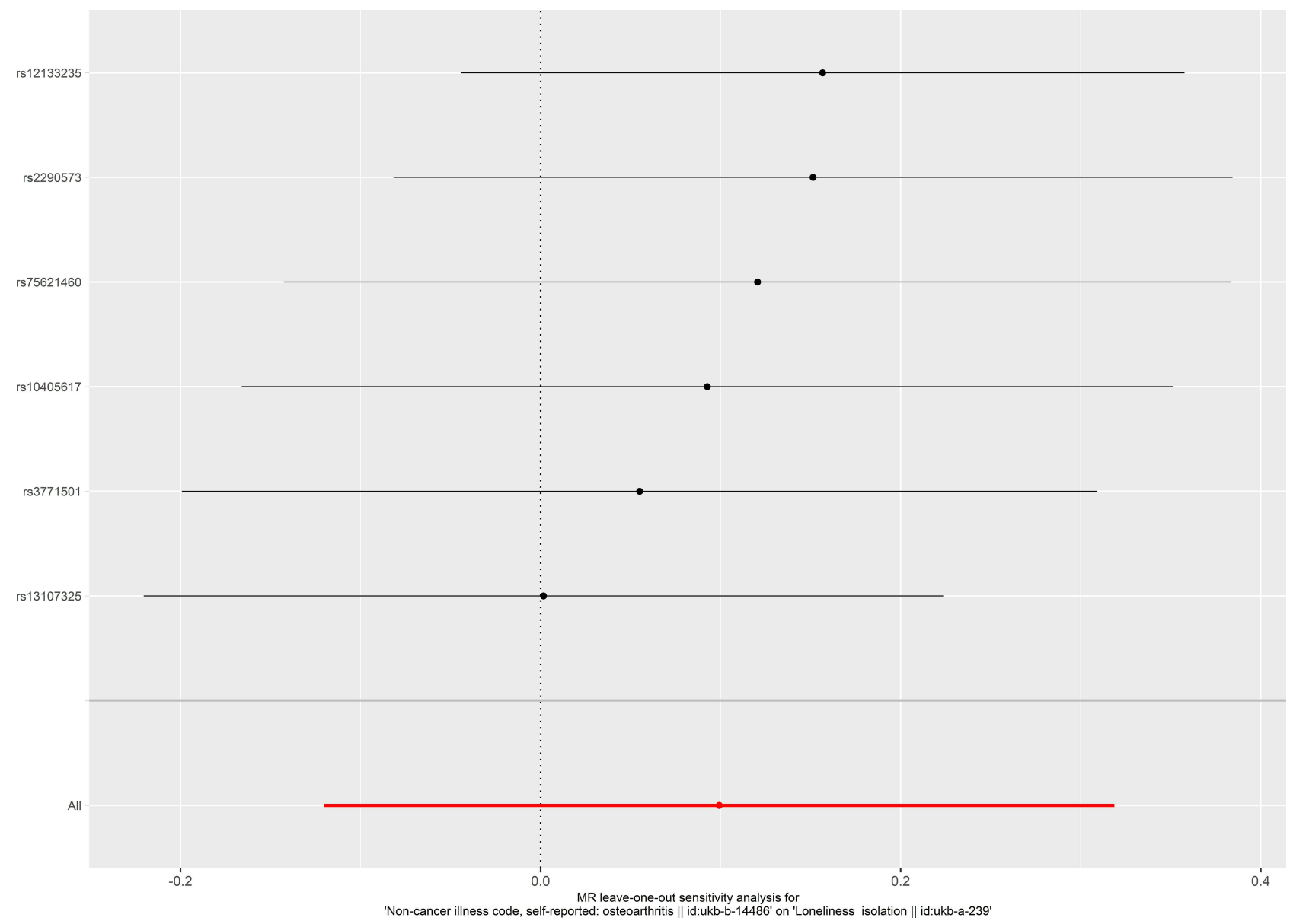

Figure 6 Leave-one-out analysis of the causal effect of osteoarthritis on social isolation. Each black point represents result of the inverse-variance weighted (IVW) method applied to estimate the causal effect of osteoarthritis on social isolation excluding each particular SNP from the analysis. Each red point depicts the IVW estimate using all SNPs. No single SNP is strongly driving the overall effect of osteoarthritis on social isolation in this leave-one-out sensitivity analysis.

\section{Conclusion}

This study showed that social isolation is causally associated with osteoarthritis. Further work is needed to investigate the underlying mechanisms and to improve the management of both conditions.

\section{Compliance with Ethical Guidelines}

This article does not include any studies with human participants or animals performed by any of the authors.

\section{Funding}

This work was supported by the Science and Technology Project of Guangzhou (201707010081) and Foundation of Guangdong (2017A030313606).

\section{Disclosure}

The authors declare that they have no competing interests.

\section{References}

1. Umberson D, Montez JK. Social relationships and health: a flashpoint for health policy. $J$ Health Soc Behav. 2010;51(1_suppl):S54-66. doi: $10.1177 / 0022146510383501$

2. Wikipedia. Social isolation; 2021. Available from: https://en.wikipe dia.org/wiki/Social_isolation. Accessed June 30, 2021.

3. Leigh-Hunt N, Bagguley D, Bash K, et al. An overview of systematic reviews on the public health consequences of social isolation and loneliness. Public Health. 2017;152:157-171. doi:10.1016/j.puhe.2017.07.035

4. Loades ME, Chatburn E, Higson-Sweeney N, et al. Rapid systematic review: the impact of social isolation and loneliness on the mental health of children and adolescents in the context of COVID-19. J Am Acad Child Adolesc Psychiatry. 2020;59(11):1218-1239 e1213. doi:10.1016/j.jaac.2020.05.009

5. Holt-Lunstad J. The potential public health relevance of social isolation and loneliness: prevalence, epidemiology, and risk factors. Public Policy Aging Rep. 2018;27(4):127-130. doi:10.1093/ppar/prx030

6. Wu B. Social isolation and loneliness among older adults in the context of COVID-19: a global challenge. Glob Health Res Policy. 2020;5(1):27. doi:10.1186/s41256-020-00154-3

7. Escalante E, Golden RL, Mason DJ. Social isolation and loneliness: imperatives for health care in a post-COVID world. JAMA. 2021;325 (6):520-521. doi:10.1001/jama.2021.0100 
8. Clair R, Gordon M, Kroon M, Reilly C. The effects of social isolation on well-being and life satisfaction during pandemic. Humanit Soc Sci. 2021;8(1):28. doi:10.1057/s41599-021-00710-3

9. Alcaraz KI, Eddens KS, Blase JL, et al. Social isolation and mortality in US Black and White men and women. Am J Epidemiol. 2019;188 (1):102-109. doi:10.1093/aje/kwy231

10. Gallagher S, Bennett KM, Roper L. Loneliness and depression in patients with cancer during COVID-19. J Psychosoc Oncol. 2021;39 (3):445-451. doi:10.1080/07347332.2020.1853653

11. Hakulinen C, Pulkki-Råback L, Virtanen M, Jokela M, Kivimäki M, Elovainio M. Social isolation and loneliness as risk factors for myocardial infarction, stroke and mortality: UK Biobank cohort study of 479054 men and women. Heart. 2018;104(18):1536-1542. doi:10.1136/heartjnl-2017-312663

12. Salinas J, Beiser A, Himali JJ, et al. Associations between social relationship measures, serum brain-derived neurotrophic factor, and risk of stroke and dementia. Alzheimers Dement. 2017;3(2):229-237. doi:10.1016/j.trci.2017.03.001

13. Kloppenburg M, Berenbaum F. Osteoarthritis year in review 2019: epidemiology and therapy. Osteoarthritis Cartilage. 2020;28 (3):242-248. doi:10.1016/j.joca.2020.01.002

14. Siviero P, Veronese N, Smith T, et al. Association between osteoarthritis and social isolation: data from the EPOSA study. J Am Geriatr Soc. 2020;68(1):87-95. doi:10.1111/jgs.16159

15. Yin J, Lassale C, Steptoe A, Cadar D. Exploring the bidirectional associations between loneliness and cognitive functioning over 10 years: the English longitudinal study of ageing. Int J Epidemiol. 2019;48(6):1937-1948. doi:10.1093/ije/dyz085

16. Emdin CA, Khera AV, Kathiresan S. Mendelian randomization. JAMA. 2017;318(19):1925-1926. doi:10.1001/jama.2017.17219

17. Hemani G, Bowden J, Davey Smith G. Evaluating the potential role of pleiotropy in Mendelian randomization studies. Hum Mol Genet. 2018;27(R2):R195-r208. doi:10.1093/hmg/ddy163

18. Davies NM, Holmes MV, Davey Smith G. Reading Mendelian randomisation studies: a guide, glossary, and checklist for clinicians. BMJ. 2018;362:k601. doi:10.1136/bmj.k601

19. Zheng J, Baird D, Borges MC, et al. Recent developments in Mendelian randomization studies. Curr Epidemiol Rep. 2017;4 (4):330-345. doi:10.1007/s40471-017-0128-6

20. Shen LX, Yang YX, Kuo K, et al. Social isolation, social interaction, and Alzheimer's disease: a Mendelian randomization study. J Alzheimers Dis. 2021;80(2):665-672. doi:10.3233/JAD-201442

21. Hemani G, Zheng J, Elsworth B, et al. The MR-Base platform supports systematic causal inference across the human phenome. Elife. 2018;7:e34408. doi:10.7554/eLife.34408
22. He Y, Zheng C, He MH, Huang JR. The causal relationship between body mass index and the risk of osteoarthritis. Int J Gen Med. 2021;14:2227-2237. doi:10.2147/IJGM.S314180

23. Bowden J, Del Greco MF, Minelli C, Davey Smith G, Sheehan N, Thompson J. A framework for the investigation of pleiotropy in two-sample summary data Mendelian randomization. Stat Med. 2017;36(11):1783-1802. doi:10.1002/sim.7221

24. Bowden J, Davey Smith G, Haycock PC, Burgess S. Consistent estimation in Mendelian randomization with some invalid instruments using a weighted median estimator. Genet Epidemiol. 2016;40(4):304-314. doi:10.1002/gepi.21965

25. Hartwig FP, Davey Smith G, Bowden J. Robust inference in summary data Mendelian randomization via the zero modal pleiotropy assumption. Int J Epidemiol. 2017;46(6):1985-1998. doi:10.1093/ije/ dyx 102

26. Burgess S, Thompson SG, Collaboration CCG. Avoiding bias from weak instruments in Mendelian randomization studies. Int J Epidemiol. 2011;40(3):755-764. doi:10.1093/ije/dyr036

27. Rezakhani Moghaddam H, Nadrian H, Abbagolizadeh $N$, Babazadeh T, Aghemiri M, Fathipour A. Mental health-ill health differences in disease severity and its sociodemographic biobehavioral predictors among patients with knee osteoarthritis. Clin Nurs Res. 2019;28(7):886-904. doi:10.1177/1054773817751527

28. Brown EG, Gallagher S, Creaven AM. Loneliness and acute stress reactivity: a systematic review of psychophysiological studies. Psychophysiology. 2018;55(5):e13031. doi:10.1111/psyp.13031

29. Santos ML, Gomes WF, Pereira DS, et al. Muscle strength, muscle balance, physical function and plasma interleukin-6 (IL-6) levels in elderly women with knee osteoarthritis (OA). Arch Gerontol Geriatr. 2011;52(3):322-326. doi:10.1016/j.archger.2010.05.009

30. Guss JD, Ziemian SN, Luna M, et al. The effects of metabolic syndrome, obesity, and the gut microbiome on load-induced osteoarthritis. Osteoarthritis Cartilage. 2019;27(1):129-139. doi:10.1016/j.joca.2018.07.020

31. Liu Y, Ding W, Wang HL, et al. Gut microbiota and obesity-associated osteoarthritis. Osteoarthritis Cartilage. 2019;27 (9):1257-1265. doi:10.1016/j.joca.2019.05.009

32. Makris AP, Karianaki M, Tsamis KI, Paschou SA. The role of the gut-brain axis in depression: endocrine, neural, and immune pathways. Hormones. 2021;20(1):1-12. doi:10.1007/s42000-02000236-4

33. Pierce BL, Burgess S. Efficient design for Mendelian randomization studies: subsample and 2-sample instrumental variable estimators. Am J Epidemiol. 2013;178(7):1177-1184. doi:10.1093/aje/kwt084
International Journal of General Medicine

\section{Publish your work in this journal}

The International Journal of General Medicine is an international, peer-reviewed open-access journal that focuses on general and internal medicine, pathogenesis, epidemiology, diagnosis, monitoring and treatment protocols. The journal is characterized by the rapid reporting of reviews, original research and clinical studies across all disease areas. The manuscript management system is completely online and includes a very quick and fair peer-review system, which is all easy to use. Visit http://www.dovepress.com/ testimonials.php to read real quotes from published authors. 